FOR THE RECORD

\title{
Novel surfactant mixtures for NMR spectroscopy of encapsulated proteins dissolved in low- viscosity fluids
}

\author{
RONALD W. PETERSON, MAXIM S. POMETUN, ZHENGSHUANG SHI, AND \\ A. JOSHUA WAND \\ Johnson Research Foundation and Department of Biochemistry and Biophysics, University of Pennsylvania, \\ Philadelphia, Pennsylvania 19104-6059, USA
}

(Received April 22, 2005; Final Revision July 22, 2005; Accepted July 22, 2005)

\begin{abstract}
NMR spectroscopy of encapsulated proteins dissolved in low-viscosity fluids is emerging as a tool for biophysical studies of proteins in atomic detail in a variety of otherwise inaccessible contexts. The central element of the approach is the encapsulation of the protein of interest within the aqueous core of a reverse micelle with high structural fidelity. The process of encapsulation is highly dependent upon the nature of the surfactant(s) employed. Here we describe novel mixtures of surfactants that are capable of successfully encapsulating a range of types of proteins under a variety of conditions.
\end{abstract}

Keywords: reverse micelle; mixed surfactant systems; NMR spectroscopy; protein encapsulation

NMR spectroscopy of encapsulated proteins dissolved in low-viscosity fluids is an emerging tool for structural biology and for biophysical studies of proteins in atomic detail in a variety of otherwise inaccessible contexts (Wand et al. 1998, 2003). For example, the scalable interior volume of the reverse micelle provides a mechanism for investigating the effects of confinement on protein stability and folding (Peterson et al. 2004) and, if appropriate surfactants are employed, can also be used to investigate the phenomenon of protein cold denaturation under otherwise native conditions (Babu et al. 2004).

A critical requirement is the need to encapsulate a protein of interest without altering its native structure. To date, only a small number of surfactant mixtures have been demonstrated to allow for high-resolution NMR studies of proteins in low-viscosity alkane solvents such as pentane, butane, propane (Wand et al. 1998), or ethane (Peterson

Reprint requests to: A. Joshua Wand, Department of Biochemistry and Biophysics, University of Pennsylvania, Philadelphia, PA 191046059, USA; e-mail: wand@mail.med.upenn.edu; fax: (215) 573-7290.

Article published online ahead of print. Article and publication date are at http://www.proteinscience.org/cgi/doi/10.1110/ps.051535405. et al. 2005). These include the anionic surfactant sodium bis(2-ethylhexyl)-sulfosuccinate (AOT) (Wand et al. 1998) and the cationic surfactant cetyltrimethylammonium bromide (CTAB) (Lefebvre et al. 2005). To achieve optimal performance for high-resolution NMR, the volume of the reverse micelle is strictly controlled by limiting the amount of water available to form the internal aqueous core. Under the condition of a limited water pool, the interaction between the protein and the surfactant head groups can potentially alter the protein structure. This undesirable effect can sometimes but not always be overcome with the addition of salts to disrupt electrostatic interactions, but this often reduces the encapsulation efficiency. Here we report the development and evaluation of novel surfactant mixtures that appear to allow considerable flexibility for NMR studies of encapsulated proteins dissolved in lowviscosity solvents.

\section{Results}

To reduce deleterious protein-surfactant interactions, we have pursued the use of nonionic surfactants and 
have focused on those having an ethylene glycol head group with a linear alkyl ether chain tail (Shioi et al. 1997; Fig. 1A). The nomenclature for this family of surfactants is $\mathrm{C}_{i} \mathrm{E}_{j}$, where $i$ represents the number of carbons in the alkyl chain, and $j$ is the number of glycol subunits in the head group. Initial tests were performed with $\mathrm{C}_{12} \mathrm{E}_{4}$, though other members of this family have also been examined with success. In order to reduce the potentially destabilizing electrostatic interactions between the surfactant and the protein but still allow for some electrostatic complementarity, a $70 \%$ nonionic/ $30 \%$ AOT mixture was found to give optimal efficiency of encapsulation (Shioi et al. 1997).

Essentially all proteins that we have previously reported as successfully encapsulated in AOT or CTAB reverse micelles dissolved in low-viscosity alkanes are also successfully encapsulated with this new formulation. Example ${ }^{15} \mathrm{~N}-\mathrm{HSQC}$ spectra for ubiquitin and flavodoxin are given in Figure 1, B and C. Both spectra exhibit very minor differences compared to aqueous solution and are also nearly identical to spectra of ubiquitin and flavodoxin in $\mathrm{AOT}$ and $\mathrm{CTAB}$ reverse micelles, respectively. Of primary interest is that the $\mathrm{C}_{12} \mathrm{E}_{4}$ /AOT system does not require the use of high viscosity cosurfactants, such as hexanol in the case for $\mathrm{CTAB}$, to induce reverse micelle formation. This is of critical importance for a surfactant system that is to be used for experiments where low viscosity is a key requirement. This is particularly true in the context of studies of protein cold-induced denaturation since controlling the viscosity at low temperatures is critical.

The $\mathrm{C}_{12} \mathrm{E}_{4}$ surfactant molecule is significantly longer than AOT. Small-angle X-ray scattering results indicate that for a water loading (the molar ratio of water to surfactant) of 10 , the $70 \% \quad \mathrm{C}_{12} \mathrm{E}_{4}: 30 \%$ AOT reverse micelle particle is about six times the volume of the AOT reverse micelle (Shioi et al. 1997). The increase in volume, while a slight disadvantage at low water loadings for small proteins, becomes less important as the protein size increases since it becomes a smaller component of the overall reverse micelle dimensions (Wand et al. 2003).

Protein encapsulation can be further optimized by the use of a triple surfactant system. Here the idea is to
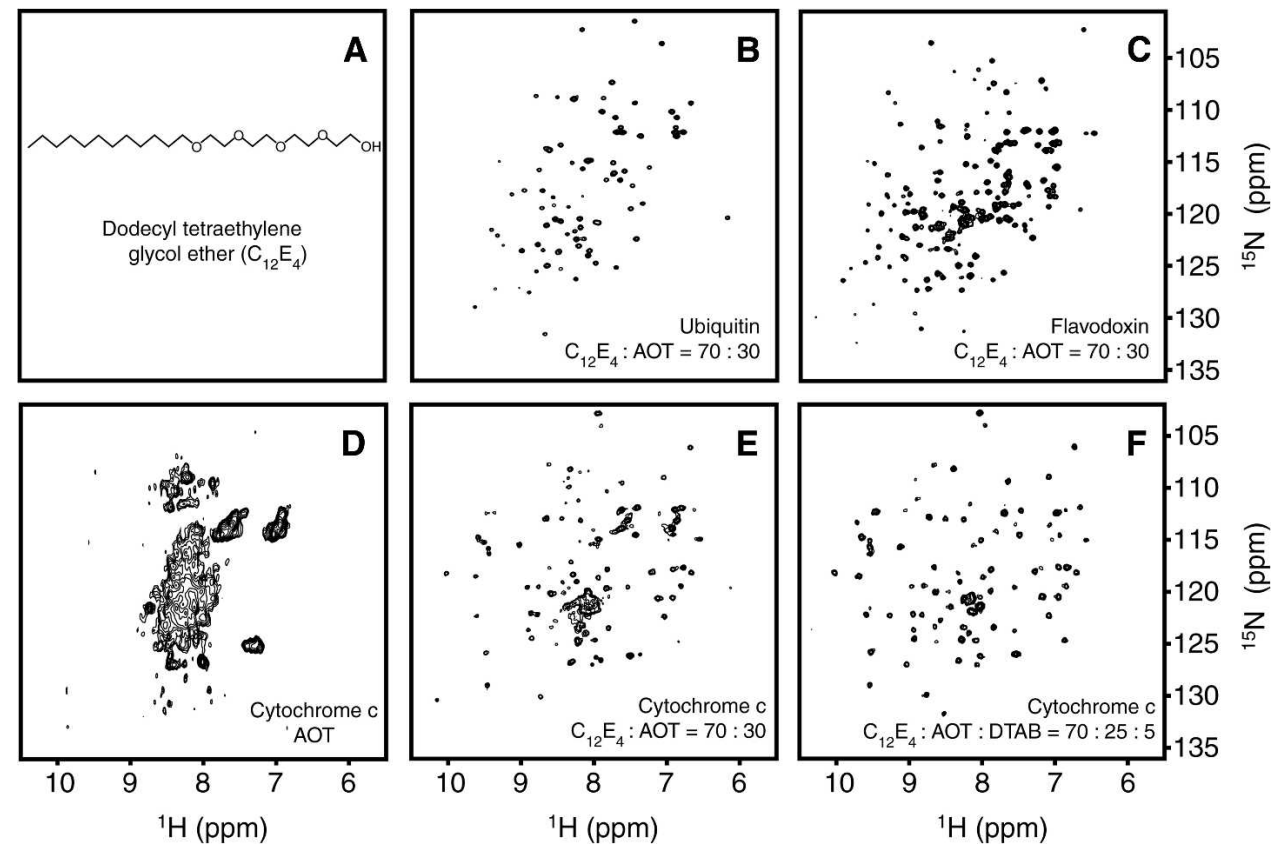

Figure 1. Performance of novel surfactant mixtures for NMR spectroscopy of encapsulated proteins dissolved in low-viscosity fluids. ( $A$ ) Nonionic surfactant $\mathrm{C}_{12} \mathrm{E}_{4}$. $(B){ }^{15} \mathrm{~N}-\mathrm{HSQC}$ spectrum of recombinant human ubiquitin (Wand et al. 1996) solubilized in $50 \mathrm{mM}$ sodium acetate $(\mathrm{pH} 5.0)$, and encapsulated with a water loading of 10 in $100 \mathrm{mM} 70 \% \mathrm{C}_{12} \mathrm{E}_{4} / 30 \%$ AOT reverse micelles in pentane. $(C){ }^{15} \mathrm{~N}$-HSQC spectrum of recombinant flavodoxin (Liu et al. 2001) solubilized in $20 \mathrm{mM}$ sodium phosphate (pH 5.8), and encapsulated with a water loading of 10 in $100 \mathrm{mM} 70 \% \mathrm{C}_{12} \mathrm{E}_{4} / 30 \%$ AOT. $(D){ }^{15} \mathrm{~N}-\mathrm{HSQC}$ spectrum of cytochrome $c$ solubilized in $50 \mathrm{mM}$ sodium acetate (pH 5.0), and encapsulated with a water loading of 10 in $100 \%$ AOT reverse micelles in pentane. $(E){ }^{15} \mathrm{~N}-\mathrm{HSQC}$ spectrum of cytochrome $c$ solubilized in $50 \mathrm{mM}$ sodium acetate (pH 5.0), and encapsulated with a water loading of 10 in $70 \% \mathrm{C}_{12} \mathrm{E}_{4} / 30 \%$ AOT reverse micelles in pentane. $(F){ }^{15} \mathrm{~N}$-HSQC spectrum of cytochrome $c$ solubilized in $50 \mathrm{mM}$ sodium acetate ( $\mathrm{pH} 5.0$ ), and encapsulated with a water loading of 10 in $70 \% \mathrm{C}_{12} \mathrm{E}_{4} / 25 \%$ $\mathrm{AOT} / 5 \%$ DTAB in pentane. Spectra were collected on a $600-\mathrm{MHz}$ Varian Inova-class spectrometer equipped with a cold probe. 
adjust the ratios of nonionic, cationic, and anionic surfactants to match the charge composition on the surface of the protein. The fraction of nonionic surfactant is adjusted to reflect the ratio of charged/uncharged residues in the protein. Model compound data are then used to provide estimates of $\mathrm{pKa}$ values in order to calculate the number of positive and negative charges on the protein at a particular $\mathrm{pH}$, and the molar ratio of cationic to anionic surfactants is adjusted accordingly. The following example using a recombinant pseudo-wildtype cytochrome $c$ (Rumbley et al. 2002) illustrates the approach.

Cytochrome $c$ is an extremely basic protein, and as a result, the electrostatic interaction with $100 \%$ AOT reverse micelles results in a highly nonnative structure, which is reflected in a collapsed ${ }^{15} \mathrm{~N}-\mathrm{HSQC}$ spectrum (Fig. 1D). To ameliorate this, the protein was encapsulated in a $70 \% \mathrm{C}_{12} \mathrm{E}_{4}: 30 \%$ AOT, which closely matches the ratio of nonionizable residues to ionizable residues in the protein sequence. Although the ${ }^{15} \mathrm{~N}-\mathrm{HSQC}$ spectrum of cytochrome $c$ in this mixture is significantly closer to the free solution spectrum, a cluster of largely unresolved cross-peaks near the center of the spectrum remains (Fig. 1E). A calculation of the total charge composition of the protein at $\mathrm{pH} 5$ suggests that the ratio of charged groups is $\sim 70 \%$ cationic and $30 \%$ anionic. To balance this charge composition, a ratio of 70:25:5 for nonionic:anionic:cationic surfactants was found to be optimal. Here we employed decyltrimethylammonium bromide (DTAB) as the cationic surfactant. In the charge-balanced surfactant system composed of $70 \% \mathrm{C}_{12} \mathrm{E}_{4}: 25 \%$ AOT:5\% DTAB the spectrum of recombinant pseudo-wild-type cytochrome $c$ (Fig. $1 \mathrm{~F}$ ) is essentially identical to the free solution spectrum, clearly indicating that the native structure is retained.

In the context of cold denaturation, it has been shown previously that AOT reverse micelles are indefinitely stable at temperatures as low as $-20^{\circ} \mathrm{C}$ and partially stable at $-30^{\circ} \mathrm{C}$ and below with a slow, continuous loss of water (Babu et al. 2004). The CTAB/hexanol surfactant system has poor tolerance to low temperature and undergoes a phase transition near $-10^{\circ} \mathrm{C}$ (Lefebvre et al. 2005), effectively eliminating it for studies of cold denaturation or for optimization of structural studies by quenching of thermal motion (Skalicky et al. 2001). Since the triple-surfactant method appears to represent a more universal approach to protein encapsulation, it was interesting to see if new surfactant mixes were suitable for cold-induced denaturation studies. The double- and triple-surfactant mixes reported herein performed well at low temperatures with cytochrome $c$.

In summary, the use of the double $\mathrm{C}_{i} \mathrm{E}_{j} / \mathrm{AOT}$ and the triple $\mathrm{C}_{i} \mathrm{E}_{j} / \mathrm{AOT} / \mathrm{DTAB}$ surfactant systems allows effective control over the charge-charge interactions and presents a robust approach to encapsulate proteins in their native state. This has all but eliminated a significant bottleneck in the reverse micelle method. It is important to note that the surfactant combinations mentioned in this paper are merely guidelines for other proteins. The precise combination of nonionic and ionic surfactants can be varied over a broad range in attempts to accommodate a new protein. By varying the ratios of ionic to nonionic and cationic to anionic surfactants in the mixture, all proteins that have been successfully encapsulated previously by this lab have been encapsulated with high structural fidelity and with little difficulty in mixtures described here. It is especially encouraging that the requirement for high viscosity cosurfactants, such as hexanol, in preparing reverse micelles can be avoided by using nonionic surfactants. Furthermore, the stability of the double and the triple surfactant reverse micelles at low temperatures makes them ideal for cold-induced unfolding studies, which now can be performed on a wide range of proteins.

\section{Acknowledgments}

This work was supported by NIH grants GM 62874 and GM 35940, and by NSF grant DMR00-79909.

\section{References}

Babu, C.R., Hilser, V.J., and Wand, A.J. 2004. Direct access to the cooperative substructure of proteins and the protein ensemble via cold denaturation. Nat. Struct. Mol. Biol. 11: 352-357.

Lefebvre, B.G., Liu, W., Peterson, R.W., Valentine, K.G., and Wand, A.J. 2005. NMR spectroscopy of proteins encapsulated in a positivelycharged surfactant. J. Magn. Res. 175: 158-162.

Liu, W., Flynn, P.F., Fuentes, E.J., Kranz, J.K., McCormick, M., and Wand, A.J. 2001. Main chain and side chain dynamics of oxidized flavodoxin from Cyanobacterium anabaena. Biochemistry 40: 14744 14753.

Peterson, R.W., Anbalagan, K., Tommos, C., and Wand, A.J. 2004. Forced folding and structural analysis of metastable proteins. J. Am. Chem. Soc. 126: 9498-9499.

Peterson, R.W., Lefebvre, B.G., and Wand, A.J. 2005. High resolution NMR studies of encapsulated proteins in liquid ethane. J. Am. Chem. Soc. 127: 10176-10177.

Rumbley, J.N., Hoang, L., and Englander, S.W. 2002. Recombinant equine cytochrome $c$ in Escherichia coli: High-level expression, characterization, and folding and assembly mutants. Biochemistry 41: 13894 13901.

Shioi, A., Harada, M., Takahashi, H., and Adachi, M. 1997. Protein extraction in a tailored reversed micellar system containing nonionic surfactants. Langmuir 13: 609-616.

Skalicky, J.J., Mills, J.L., Sharma, S., and Szyperski, T. 2001. Aromatic ring-flipping in supercooled water: Implications for NMR-based structural biology of proteins. J. Am. Chem. Soc. 123: 388-397.

Wand, A.J., Urbauer, J.L., McEvoy, R.P., and Bieber, R.J. 1996. Internal dynamics of human ubiquitin revealed by ${ }^{13} \mathrm{C}$-relaxation studies of randomly fractionally labeled protein. Biochemistry 35: 6116-6125.

Wand, A.J., Ehrhardt, M.R., and Flynn, P.F. 1998. High-resolution NMR of encapsulated proteins dissolved in low-viscosity fluids. Proc. Natl. Acad. Sci. 95: 15299-15302.

Wand, A.J., Babu, C.R., Flynn, P.F., and Milton, M.J. 2003. NMR spectroscopy of encapsulated proteins dissolved in low viscosity fluids. Biol. Magn. Reson. 20: 121-160. 